\title{
Correction to: Racial Constructs in Youth Mentoring: Reconsidering Race and Ethnicity
}

\section{Correction to:}

Chapter 4 in: A. J. Hoffman et al., The Role of Community Development in Reducing Extremism and Ethnic Conflict, https://doi.org/10.1007/978-3-319-75699-8_4

The original version of the book was published without a reference citation in this chapter. The citation has been included in this revised version.

The updated original online version of this chapter can be found at https://doi.org/10.1007/978-3-319-75699-8_4 